\section{The continuing tale of cytokeratins in Barrett's mucosa: As you like it}

Barrett's adenocarcinoma (BA) has seen a rapid increase in incidence throughout the Western world. The diagnosis of BA is often at an advanced stage and is generally associated with a poor prognosis and a mean survival of less than one year. Adenocarcinomas however do not arise de novo but follow an established sequence from Barrett's metaplasia (BM) through dysplasia to neoplasia.

Efforts to intervene in the pathogenesis of oesophageal adenocarcinomas have so far been disappointing. Reduction of gastro-oesophageal reflux disease has led to minimal regression of $\mathrm{BM}$ and has yet to be shown to have any impact on cancer prevention. Surveillance programmes for patients with $\mathrm{BM}$ have had variable results and have raised important questions about their cost effectiveness and of better risk stratification of patients with BM. The prevalence of $\mathrm{BM}$ in the general population is approximately $1-3 \%$, with only $0.5-1 \%$ of patients with BM converting to neoplasia each year. ${ }^{12}$ The reliable diagnoses of intestinal metaplasia and dysplasia have also been difficult to validate in each patient, mostly related to sampling errors due to the variable anatomy of the lower oesophagus and their patchy distribution within a segment of BM.

Our understanding of the molecular biology of BM has yielded many phenotypic and genetic changes within the epithelium that are associated with different stages along the metaplasia-dysplasia-neoplasia sequence. ${ }^{3}$ It has been suggested that some of these changes, such as cytokeratin subsets, might aid in the diagnosis and management of patients with $\mathrm{BM}$ and is the focus of the paper by Couvelard et al in this issue of Gut (See page 761). ${ }^{4}$
Cytokeratins are highly conserved polypeptides that heterodimerise and form the building blocks for the intermediate filaments as part of the cell cytoskeleton. Intermediate filaments are anchored to desmosomes in the cell membrane and are of particular interest in epithelial systems in maintaining cell morphology, polarity, and intercellular adhesion. ${ }^{5}$ Cytokeratins are expressed in 20 distinct forms in epithelial cells but are absent from mesenchymal tissue where vimentin is used in the assembly of intermediate filaments. ${ }^{5}$

There are variable patterns of expression of cytokeratins in epithelial cells depending on the type, location, and differentiation of epithelium. Some cytokeratins have a broad range of expression in columnar epithelium, such as CK8 and CK19, while others such as CK7 and CK20 show highly restricted expression. ${ }^{6} \mathrm{CK} 20$ is commonly used as a marker of intestinal differentiation. It is expressed on the surface and crypt epithelium of the normal colon and small intestine. In the stomach, expression is limited to the surface foveolar epithelium, with no gastric gland or pit staining. CK7 has been proposed as a marker of ductal differentiation. It is expressed in ductal breast carcinomas but not in the normal epithelium of the gastrointestinal tract. Cytokeratins are very stable proteins and are conserved in most epithelial cancers, even in the presence of gross phenotypic and genetic alterations. For this reason, detection of cytokeratin subsets such as CK7 and CK20 have become very useful in diagnosing the origins of occult or poorly differentiated cancers and are part of current clinical practice.

Differential patterns of cytokeratin expression have been demonstrated in the oesophagus and proposed as useful clinical markers. A phenotypic map of cytokeratin expression validates our current histopathological categorisation of the gastro-oesophageal junction (fig 1). However, a unique pattern of CK7 and CK20 immunohistochemical

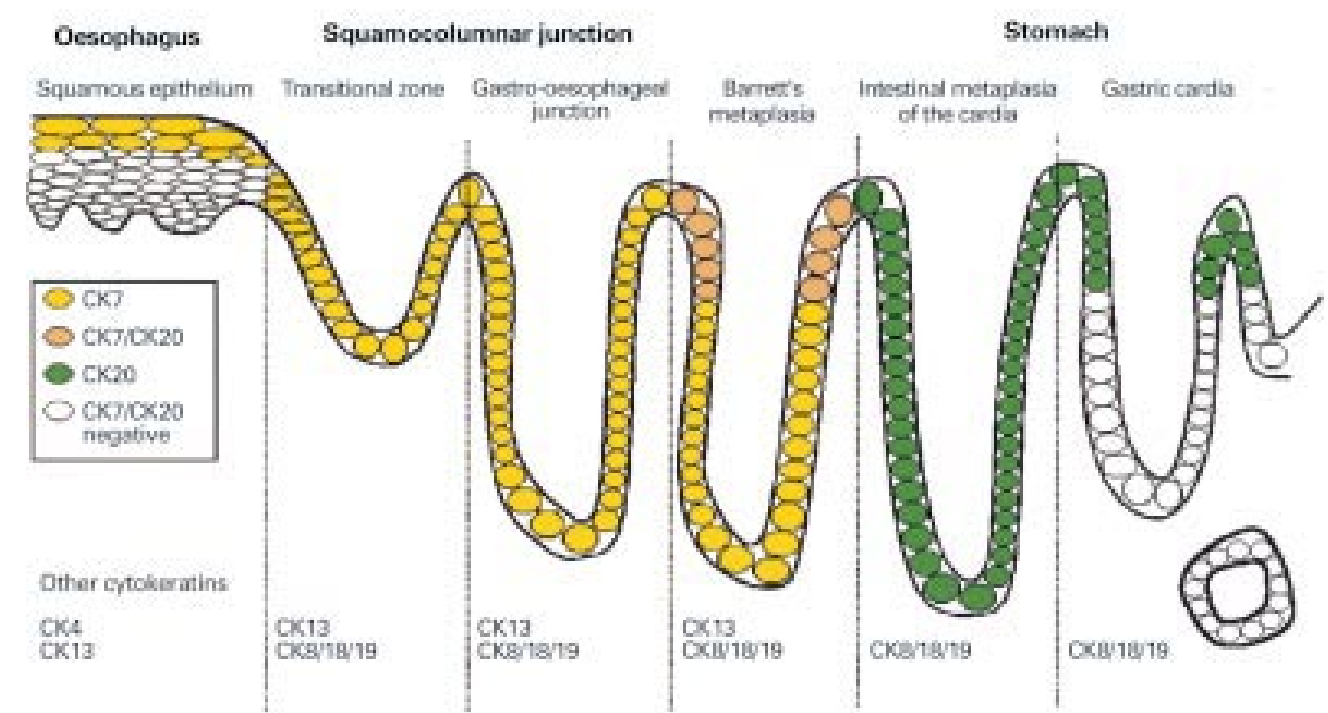

Figure 1 Schematic illustration of cytokeratin (CK) 7 and 20 expression in the normal oesophagus, Barrett's metaplasia, squamocolumnar junction, and proximal stomach. CK 7 is only found in the superficial layers of the squamous epithelium in contrast with columnar lined tissue in the oesophagus that shows increased expression throughout the crypts and glands. CK20 is limited to superficial areas of Barrett's metaplasia and the gastric cardia but upregulated in intestinal metaplasia of the cardia. 
expression, designated the BM CK7/20 pattern, has been demonstrated to be both sensitive and specific to Barrett's oesphagus and may be used as an objective marker of BM. ${ }^{7}$ This pattern shows superficial CK20 staining and strong CK7 staining of both superficial and deep glands and can be used to distinguish BM from intestinal metaplasia of the stomach which, although can be histologically indistinguishable, has a different pattern of cytokeratin expression, is associated with Helicobacter pylori infection, and is not associated with an increased risk of malignancy. ${ }^{8}$

The Barrett's CK7/20 pattern has been demonstrated in both long and short segment BM. The paper by Couvelard et al confirms the presence of a Barrett's CK7/20 pattern and demonstrates that the same CK7/20 pattern holds true for ultrashort segment BM. ${ }^{4}$ The authors further suggest that these findings may be useful to improve endoscopic surveillance strategies to specifically target those at increased risk of $\mathrm{BM}$ and its complications. Ultrashort BM is however an area surrounded by controversy. It is defined as intestinal metaplasia found at the gastro-oesophageal junction arising in either very short tongues of columnar mucosa or in eccentric or normal appearing squamocolumnar junctions (SCJs). It has been reported that the prevalence of this may be up to $43 \%$ in unselected patients undergoing routine endoscopy. The nature of metaplasia in eccentric or normal SCJs remains unknown. This has prompted much debate as to the risk (if any) of dysplasia or indeed cancer in this group and the appropriateness of endoscopic surveillance. Furthermore, if we are to regard this lesion in a similar way to short and long segment Barrett's and it is found in up to $43 \%$ of normal appearing SCJs, should we be performing screening biopsies of these patients? Certainly this does not seem to be a plausible option.

Perhaps it is in the understanding of the biology of BM where cytokeratins have already made a potentially useful contribution. Specifically, rearrangements in the composition of lateral cell-cell adhesion junctions, the desmosomes, have been recently reported in BM. ${ }^{9}$ These alterations in adhesion complexes while being associated with concomitant changes in cytokeratins are also important in releasing gamma catenin, a known oncogene involved in T cell factor/ leucocyte enhancing factor transcription. ${ }^{10}$

In conclusion, greater understanding of the processes involved in the development and progression of BM is crucial if we are to develop strategies to intervene at an earlier stage in the metaplasia-dysplasia-carcinoma sequence and alter the outcome of oesophageal adenocarcinomas. Cytokeratins are undoubtedly of great scientific importance but caution should be observed in areas where clinical risk has not been already established.

A LATCHFORD B EKSTEEN

Epithelial Laboratory, Division of Medical Sciences, J JANKOWSKI

University of Birmingham, UK

jjankowski@bham.ac.uk

1 Jankowski JA, Perry I, Harrison RF. Gastro-oesophageal cancer: death at the junction. BMf 2000;321:463-4

2 Jankowski JA, Harrison RF, Perry I, et al. Barrett's metaplasia. Lancet 2000; 356:2079-85.

3 Jankowski JA, Wright NA, Meltzer SJ, et al. Molecular evolution of the metaplasia-dysplasia-adenocarcinoma sequence in the esophagus. Am $\mathcal{F}$ Pathol 1999;154:965-73.

4 Couvelard A, Cauvin J-M, Goldfain D, et al. Cytokeratin immunoreactivity of intestinal metaplasia at normal oesophagogastric junction indicates its aetiology. Gut 2001;49:761-6.

5 Moll R, Franke WW, Schiller DL, et al. The catalog of human cytokeratins: patterns of expression in normal epithelia, tumors and cultured cells. Cell 1982;31:11-24.

6 Moll R, Lowe A, Laufer J, et al. Cytokeratin 20 in human carcinomas. A new histodiagnostic marker detected by monoclonal antibodies. Am $\mathcal{F}$ Pathol 0:427-47.

7 Ormsby AH, Goldblum JR, Rice TW, et al. Cytokeratin subsets can reliably distinguish Barrett's esophagus from intestinal metaplasia of the stomach. Hum Pathol 1999;30:288-94.

8 Ormsby AH, Vaezi MF, Richter JE, et al. Cytokeratin immunoreactivity patterns in the diagnosis of short-segment Barrett's esophagus. Gastroenterology 2000;119:683-90.

9 Morris CD, Tselepis C, Harrison RF, et al. Abnormal expression and function of desmosomal cadherins in the neoplastic progression of Barrett's oesophagus. Gastroenterology 2001;120:A425.

10 Bailey T, Biddlestone L, Shepherd N, et al. Altered cadherin and catenin complexes in the Barrett's esophagus-dysplasia-adenocarcinoma sequence: correlation with disease progression and dedifferentiation. Am $\mathcal{F}$ Pathol 1998;152:135-44.

\section{Monocytes or T cells in Crohn's disease: does IL-16 allow both to play at that game?}

Interleukin (IL)-16 was first described in 1982 under the name "lymphocyte chemoattractant factor". "Since its cloning in $1994,{ }^{2}$ the complex structure and biological function of this cytokine has been extensively explored. In 1999, the IL-16 gene was localised to chromosome $15 \mathrm{q} 26.3^{3}$ but the role of genetic variants of this gene have yet to be explored in human disease.

IL-16 can be produced by a variety of inflammatory cells, including mast cells, eosinophils, mononuclear phagocytes, and CD4+ and CD8+ $\mathrm{T}$ cells. ${ }^{4} \mathrm{IL}-16$ is expressed as an $80 \mathrm{kDa}$ precursor molecule, ${ }^{5}$ which is processed to active IL-16 by caspase $3 .{ }^{6}$

Most interestingly, the main receptor for IL-16 appears to be the CD4 molecule (which identifies T helper cells but is also present on monocytes and other phagocytes). It is assumed that interaction with the CD4 molecule is the main event in induction of most IL-16 mediated biological effects although other receptors and co-receptors may exist. The main biological function of IL-16 appears to be recruitment of CD4+ T cells. In addition, IL-16 induces the production of proinflammatory cytokines (that is, tumour necrosis factor, IL-1 $\beta$, IL- 6 , and IL-15 by monocytes) ${ }^{7}$ and can regulate RAG gene expression in CD4+ B cells. The mechanisms of signal transduction of IL-16 are still unclear but appear to involve tyrosine kinases (that is, $\mathrm{p} 56 \mathrm{lck}$ ) in $\mathrm{T}$ cells, the stress activated protein kinases (SAPK) pathway, and activation of the p38 mitogen activated protein kinase (MAPK). ${ }^{89}$

Not surprisingly, IL-16 is implicated in the pathophysiology of chronic immune diseases, including allergen induced bronchial asthma, rheumatoid arthritis, ${ }^{10}$ and Crohn's disease. ${ }^{11}{ }^{12}$ It has been found to be elevated in both Crohn's disease and ulcerative colitis where a positive correlation between disease activity and IL-16 expression has been found..$^{12}$ Expression of IL-16 was also upregulated in an animal model of chronic intestinal inflammation and blocking IL-16 activity ameliorates TNBS colitis. ${ }^{11}$

In this issue of Gut, Middel and colleagues ${ }^{13}$ analysed the contribution of IL-16 to the pathophysiology of inflammatory bowel disease in an elegant study using a variety of 
molecular techniques (see page 795). They found that increased production of IL-16 is particularly important in the pathophysiology of the inflammatory lesions in Crohn's disease in comparison with ulcerative colitis. A strong relationship between increased expression of IL-16 in T cells/ mast cells and numbers of CD4+ T cells was found. Discrete accumulation of mast cells as well as increased numbers of eosinophils have frequently been seen by pathologists although their contribution to pathophysiology was unexplained until now. The role of these cells in the pathophysiology of Crohn's disease is now explained as important contributors of IL-16.

Recently, three studies have independently identified mutations in the NOD2 gene on chromosome $16 \mathrm{q}$ as a primary cause of Crohn's disease in a subset (up to $25 \%$ ) of patients. ${ }^{14-16}$ NOD2 is only expressed by monocytes/ macrophages and its role in other phagocytes is presently unclear. However, it is not expressed in lymphocytes or epithelial cells. It is currently hypothesised that a mutation in the NOD2 gene impacts the handling of bacterial pathogens by monocytes by alteration in the lipopolysaccharide/NOD2 induced activation of nuclear factor kappa $\mathrm{B}(\mathrm{NF} \kappa \mathrm{B})$. It is thought that this constitutive defect predisposes patients with Crohn's disease to chronic inflammation, which may be triggered by the commensal flora.

The argument can be made that T cells and hence IL-16 may only play a secondary role in the disease process. The study presented by Middle et al links activation of phagocytes in the pathophysiology of Crohn's disease to strong evidence of a $\mathrm{T}$ cell contribution to disease pathophysiology. Increased activation of mononuclear phagocytes that results in the release of proinflammatory cytokines and also IL-16 is directly associated with CD4+ $\mathrm{T}$ cell recruitment. As first reports showed increased activation of $\mathrm{p} 38$ MAPK in Crohn's disease, ${ }^{17}$ which may result in increased levels of IL-16, additional molecular data provided by Middle et al strongly supports this interpretation.

IL-16 may be an interesting target to interrupt the loop between mononuclear phagocytes and $\mathrm{T}$ cell activation. An important question will be whether mast cells, eosinophils, and other phagocytes also express genes of the NOD2 family. As expression of IL-16 is most likely controlled by $\mathrm{NF} \kappa \mathrm{B}$, this cytokine may be a central player in linking the different aspects of Crohn's disease pathophysiology.

Department of General Internal Medicine,

S SCHREIBER

Christian-Albrechts-University, Kiel, Germany

1 Center DM, Cruikshank WW. Modulation of lymphocyte migration by human lymphokines. I. Identification and characterization of chemoattractant activity for lymphocytes from mitogen-stimulated mononuclear cells. F Immunol 1982;128:2563-8.

2 Cruikshank WW, Center DM, Nisar N, et al. Molecular and functional analysis of a lymphocyte chemoattractant factor: Association of biologic analysis of a lymocyte chemoattractant factor: Association of biologic

3 Kim HS. Assignment of human interleukin 16 (IL16) to chromosome 15q26.3 by radiation hybrid mapping. Cytogenet Cell Genet 1999;84:93.

4 Chupp GL, Wright EA, Wu D, et al. Tissue and T cell distribution of precursor and mature IL-16. F Immunol 1998;161:3114-19.

5 Baier M, Bannert N, Werner A, et al. Molecular cloning, sequence, expression, and processing of the interleukin 16 precursor. Proc Natl Acad Sci USA 1997;94:5273-7.

6 Zhang Y, Center DM, Wu DM, et al. Processing and activation of pro-interleukin-16 by caspase 3. F Biol Chem 1998;273:1144-9.

7 Mathy NL, Scheuer W, Lanzendorfer M, et al. Interleukin-16 stimulates the expression and production of pro-inflammatory cytokines by human monocytes. Immunology 2000;100:63-9.

8 Ryan TC, Cruikshank WW, Kornfeld H, et al. The CD4-associated tyrosine kinase p56lck is required for lymphocyte chemoattractant factor-induced T kinase p56lck is required for lymphocyte chemoattractant
lymphocyte migration. $\mathcal{F}$ Biol Chem 1995;270:17081-6.

9 Krautwald S. IL-16 activates the SAPK signaling pathway in CD4+ macrophages. F Immunol 1998;160:5874-9.

10 Blaschke S, Schulz H, Schwarz G, et al. Interleukin 16 expression in relation to disease activity in rheumatoid arthritis. F Rheumatol 2001;28:12-21.

11 Keates AC, Castagliuolo I, Cruickshank WW, et al. Interleukin 16 is up-regulated in Crohn's disease and participates in TNBS colitis in mice. Gastroenterology 2000;119:972-82.

12 Seegert D, Rosenstiel P, Pfahler H, et al. Increased expression of IL-16 in inflammatory bowel disease. Gut 2001;48:326-32.

13 Middel P, Reich K, Polzien F, et al. Interleukin 16 expression and phenotype of interleukin 16 producing cells in Crohn's disease. Gut 2001;49:795-803.

14 Hugot JP, Chamaillard M, Zouali $\mathrm{H}$, et al. Association of NOD2 leucine-rich repeat variants with susceptibility to Crohn's disease. Nature 2001;411: 599-603.

15 Ogura Y, Bonen DK, Inohara N, et al. A frameshift mutation in NOD2 associated with susceptibility to Crohn's disease. Nature 2001;411:603-6.

16 Hampe J, Cuthbert A, Croucher PJP, et al. An insertion mutation in the NOD2 gene predisposes to Crohn's disease in the German and British populations. Lancet 2001;357:1925-8.

17 Waetzig GH, Seegert D, Nikolaus S, et al. Differential activity and protein expression of mitogen-activated protein kinases in inflammatory bowel disease. Gastroenterology 2001;120:A522.

\section{Sodium in preascitic cirrhosis: please pass the salt}

The relationship between sodium retention, hyperactivity of the neurohumoral vasoactive systems, and ascites formation in cirrhosis is intriguing and still remains a subject of interest and debate. According to the most widely accepted theory, the so-called peripheral arteriolar vasodilatation hypothesis of sodium retention and ascites formation in cirrhosis, the predominant mechanism in the pathogenesis of these abnormalities is the presence of persistent systemic arterial vasodilation leading to arterial hypotension, low peripheral resistance, high cardiac output, and decreased effective arterial blood volume. ${ }^{1}$ These circulatory abnormalities are detected by arterial and cardiopulmonary baroreceptors which in turn initiate the homeostatic activation of the endogenous neurohumoral systems aimed at maintaining arterial pressure within normal or near normal levels. In the kidneys however homeostatic activation of the vasoactive and sodium retaining systems promotes tubular sodium reabsorption and sodium retention. ${ }^{1}$ Because the splanchnic vasculature is a major site of arteriolar vasodilatation in cirrhosis, it is not surprising that extravasation of the fluid retained by the kidneys occurs mainly in this compartment, leading to the formation of ascites.

The renin-angiotensin-aldosterone (RAAS) and sympathetic nervous (SNS) systems, together with atrial natriuretic peptide (ANP), are the main endogenous neurohumoral systems involved in sodium homeostasis. The RAAS is one of the most extensively investigated endogenous vasoactive systems in cirrhosis because it is markedly activated in patients with sodium retention. ${ }^{2}{ }^{3}$ Plasma renin activity and plasma aldosterone levels closely correlate with urinary sodium excretion and in many decompensated cirrhotic patients they reach extraordinarily high values, with levels being higher in patients with marked sodium retention. ${ }^{23}$ The SNS is another key factor involved in the pathogenesis of sodium retention and ascites formation in cirrhosis. In fact, noradrenaline concentrations are consistently found to be increased in patients with sodium retention and ascites. ${ }^{23}$ On the other hand, endogenous natriuretic substances such 
as ANP inhibit tubular sodium reabsorption and counteract the antinatriuretic and renal vasoconstrictor effects of the RAAS and SNS. ${ }^{2}$

Whereas in decompensated cirrhosis the mechanisms underlying sodium retention are quite well established, in preascitic cirrhosis this question remains elusive. Patients with preascitic cirrhosis (patients without a history of ascites or diuretic use) usually do not exhibit sodium retention. ${ }^{2}$ Moreover, the activities of the RAAS and SNS, estimated by plasma renin activity and circulating levels of aldosterone and noradrenaline, respectively, are consistently found to be either normal or decreased in preascitic cirrhotic patients. ${ }^{2}$ These patients however present subtle abnormalities in renal sodium handling leading to an expanded circulatory blood volume but without causing ascites or oedema. Indeed, the existence of an altered renal sodium metabolism in preascitic cirrhotic patients can be readily uncovered by administering a sodium overload or following mineralocorticoid treatment. ${ }^{2}$ Therefore, and although not clinically evident, preascitic cirrhotic patients encounter a positive sodium balance which presumably is a homeostatic mechanism to compensate for the reduced effective arterial blood volume due to arteriolar vasodilation present in this condition.

In this issue of $G u t^{4}$, Wong et al examine the status of sodium homeostasis in preascitic cirrhosis by investigating renal sodium handling in 16 biopsy proven cirrhotic patients without ascites submitted to a high sodium diet (200 mmol/day) for five weeks (see page 847). The results obtained show that daily sodium intake of $200 \mathrm{mmol}$ results in weight gain and a positive sodium balance for three weeks, returning to a complete sodium balance thereafter. These findings indicate that despite continued high sodium intake, preascitic cirrhotic patients eventually reach a new steady state of sodium balance thereby preventing fluid retention and the development of ascites. As significant suppression of the RAAS and SNS, as estimated by plasma renin activity and aldosterone and noradrenaline levels, respectively, is observed in preascitic cirrhotic patients submitted to a high sodium diet for more than three weeks, the new state of sodium balance is presumably reached at the expense of intravascular volume expansion. In fact, elevated plasma ANP levels, a surrogate marker of an expanded blood volume, is consistently found in these patients during the course of the study. Interestingly, preascitic cirrhotic patients do not exhibit renal resistance to the diuretic and natriuretic effects of ANP, and thus increased plasma levels of ANP apparently contribute to the establishment of a new sodium balance in this condition. This scenario is completely different to that usually found in decompensated cirrhotic patients who show marked sodium retention despite the existence of elevated ANP plasma levels. ${ }^{5}{ }^{6}$ Finally, it is noteworthy that at the end of the study period (that is, after five weeks on a high sodium diet), when patients have already reached a new steady state level of sodium balance and plasma renin activity and plasma aldosterone levels do not differ significantly from baseline, noradrenaline levels still remain suppressed. This is not consistent with previous investigations by the same group reporting that plasma noradrenaline levels are not suppressed in preascitic cirrhotic patients submitted to an oral sodium load of $200 \mathrm{mmol} /$ day for one week. ${ }^{67}$ These controversial results could be interpreted as that evaluation of sodium homeostasis in cirrhosis without ascites is best performed over extended study periods rather than following acute or short term treatments.

In summary, the study by Wong and colleagues ${ }^{4}$ indicates that preascitic cirrhotic patients retain sodium when challenged with a high sodium intake. However, at this stage of disease, cirrhotic patients are still able to re-establish a new steady state of sodium balance mainly due to elevated levels of ANP and inhibition of the RAAS and SNS, thus preventing further development of sodium retention and ascites formation. Altogether these observations contribute to a significant advancement in our knowledge on the mechanisms underlying the disturbed sodium homeostasis of patients with preascitic cirrhosis.

J CLÀRIA

J RODÉS

Liver Unit, Hospital Clinic,

Institut d'Investigacions Biomèdiques August Pi i Sunyer (IDIBAPS),

Universitat de Barcelona, Barcelona, Spain.

1 Schrier RW, Arroyo V, Bernardi M, et al. Peripheral arteriolar vasodilation hypothesis: A proposal for the initiation of renal sodium and water retention in cirrhosis. Hepatology $1988 ; 8: 1151-7$

2 Ginès P, Rodés J. Clinical disorders of renal function in cirrhosis with ascites. In: Arroyo V, Schrier RW, Rodés J, et al, eds. Ascites and renal dysfunction in liver disease. Pathogenesis, diagnosis and treatment. Malden MA: Blackwell Science Inc, 1999:36-62.

3 Arroyo V, Planas R, Gaya J, et al. Sympathetic nervous activity, renin-angiotensin system and renal excretion of prostaglandin $\mathrm{E}_{2}$ in cirrhosis. Relationship to functional renal failure and sodium and water excretion. Eur f Clin Invest 1983;13:271-8.

4 Wong F, Liu P, Blendis L. Sodium homeostasis with chronic sodium loading in preascitic cirrhosis. Gut 2001;49:847-51.

5 Ginés P, Jiménez W, Arroyo V, et al. Atrial natriuretic factor in cirrhosis with ascites: Plasma levels, cardiac release and splanchnic extraction. Hepatology ascites: Plasma

6 Warner LC, Campbell PJ, Morali GA, et al. The response of atrial natriuretic factor and sodium excretion to dietary sodium challenges in patients with chronic liver disease. Hepatology 1990;12:460-6.

7 Wong F, Liu P, Allidina Y, et al. Pattern of sodium handling and its consequences in pre-ascitic cirrhosis. Gastroenterology 1995;108:1820-7. 